\title{
Purification of the COP9 Signalosome Complex and Binding Partners from Human T Cells
}

\author{
Aleksandr Stotland, Laura Pruitt, Phillip Webster, and Roland Wolkowicz
}

\begin{abstract}
The COP9 Signalosome (CSN) is a highly conserved eight subunit protein complex associated with a wide range of essential biological functions in eukaryotic cells, and directly involved in processes including deneddylation, phosphorylation, and ubiquitination. Despite its significant role, very few studies have been undertaken to reveal the interactions between the CSN and its binding partners, and none in human T cells. Here we present a purification method for the CSN and binding proteins via the Streptavidin-Binding Peptide (SBP) fused to CSN Subunit 1 (CSN1). Using this method, coupled with liquid chromatography-mass spectrometry analysis, we identified all eight subunits of the CSN, as well as expected and putative novel binding partners such as a tumor suppressor under the control of Cullin4a-ligase complex; Neurofibromin 2 (Merlin). This work presents a method for fast, reliable, and specific affinity-based purification of a protein complex from a nonadherent cell line. The purification of the CSN and binding partners from $\mathrm{T}$ cells can elucidate the roles of CSN in a cell type where it has never been studied before. This proteomic-based approach can broaden our understanding of the functions of the CSN in contexts such as viral-host interactions or immune activation in their natural milieu.
\end{abstract}

\section{Introduction}

$\mathbf{T}$ He COP9 Signalosome (CSN) is an eight-subunit complex originally discovered in Arabidopsis thaliana whose main function was determined to be the control of light-regulated genes in plant development (Wei and Deng, 1992). The complex is highly conserved in all living organisms, and its subunits share homology with the 26S Proteasome and the translation initiation factor 3 (eIF3), with which they can form minicomplexes of currently unknown function (Karniol et al., 1998; Schwechheimer 2004, Seeger et al., 1998). The CSN has several important roles in the eukaryotic cell, the best understood and studied of which is protein degradation via control of the deneddylation of cullins (Wei and Deng, 2003). Briefly, when a protein is targeted for degradation by the proteasomal machinery, it is marked for destruction by the addition of ubiquitin molecules by SCF ligase complexes. The complex consists of a cullin protein CUL1, a RING-finger protein Rbx1, Skp1, and an F-box protein that recruits the target and confers specificity to the scaffold (Deshaies, 1999). The activity of the SCF complex is modulated by the level of neddylation of the CUL1 protein under the CSN control. CSN removes the Nedd8 molecules from CUL1 through the metalloprotease activity of subunit 5 (CSN5), resulting in the dissociation of the Skp1/Skp2 complex from the SCF. The result of this dissociation is the binding of the TBPinteracting protein p120 (CAND1) to deneddylated CUL1, preventing the binding and ubiquitination of substrates by the SCF complex (Cope et al., 2002; Wei and Deng, 2003; Zheng et al., 2002). The ability of the CSN to negatively regulate the ubiquitin-dependent proteasomal machinery is used by the cell to regulate the levels of cell-cycle proteins such as CDK inhibitor p $27^{\mathrm{kip} 1}$, NF- $\kappa \mathrm{B}$ inhibitor $\mathrm{I} \kappa \mathrm{B}$, and HIF- $1 \alpha$ (Miyauchi et al., 2008; Morimoto et al., 2000; Read et al., 2000).

Another important function of the CSN complex performed either as a whole or by independent subunits, is the association with kinase activity. Several targets are known to be phosphorylated by the CSN-associated kinases, some of which are yet to be discovered, at least in vitro. Those targets include c-Jun, $\mathrm{I} \kappa \mathrm{B} \alpha, \mathrm{NF}-\kappa \mathrm{B}$ p105 precursor, and most importantly p53 (Bech-Otschir et al., 2001; Seeger et al., 1998). Furthermore, several of the CSN subunits, specifically CSN2 and CSN7, are known to be phosphorylated by CKII and PKD kinases with yet-to-be-discovered consequences (Uhle et al., 2003). The best characterized role of individual CSN subunits is that of subunit 5 (CSN5) in Jun signaling and AP-1 activation. CSN5, also known as Jun-activating binding protein 1 (Jab1), directly interacts with c-Jun and facilitates its binding

Department of Biology, San Diego State University, San Diego, California. 
to JNK kinase, resulting in phosphorylation and stabilization of c-Jun upon binding to AP-1 promoter sites (Claret et al., 1996; Kleemann et al., 2000).

The findings to date indicate that CSN is a major player in most cell processes, and yet only one wide-ranging proteomic study analyzing the binding partners of the CSN by liquid chromatography/mass spectrometry has been undertaken (Fang et al., 2008). Furthermore, virtually nothing is known about the interaction of the CSN in T cells with the ubiquitin ligase machinery or other proteins. This knowledge will facilitate the elucidation of how these interactions are affected by processes such as stimulation or viral infection. In order to address this question, we have purified the entire CSN from a human T-cell line through one of its subunits utilizing the Streptavidin-Binding Peptide (SBP) tag. Purification of the CSN and binding partners was further analyzed by liquid chromatography-mass spectrometry.

\section{Materials and Methods}

\section{Cells}

Human T-cell line SupT1 was obtained from the American Type Culture Collection (ATCC, Manassas, VA). Cells were maintained in complete RPMI 1640 media supplemented with $10 \%$ fetal bovine serum (Gemini Bio-Products, West Sacramento, CA), glutamine $(2 \mathrm{mM})$, penicillin $\mathrm{G}(100$ units $/ \mathrm{mL})$, and streptomycin $(100 \mu \mathrm{g} / \mathrm{mL})$. Phoenix GP cell-line (Nolan Lab, Stanford University, CA) was maintained in Dulbecco's Modified Eagle's media supplemented with $10 \%$ fetal bovine serum (Gemini Bio-Products), glutamine $(2 \mathrm{mM})$, penicillin $\mathrm{G}$ (100 units $/ \mathrm{mL})$, and streptomycin $(100 \mu \mathrm{g} / \mathrm{mL})$.

\section{Antibodies and reagents}

The antibodies to CSN1 (A300-026A), CSN2 (A300-027A), CSN4 (A300-014A), CSN7b (A300-240A), and Cullin-4a (A300-738A) were obtained from Bethyl Laboratories (Montgomery, TX). Antibodies to CSN3 (sc-100693), CSN6 (sc137122), and Merlin (sc-55575) were obtained from Santa Cruz Biotechnology, Inc (Santa Cruz, CA). The CSN8 antibody (BML-PW8290-0025) was provided by Biomol, Inc. (Plymouth Meeting, PA). The antibody for Nedd8 (2745) was obtained from Cell Signaling (Beverly, MA). The antimouse IgG-HRP (115-035-003) and antirabbit IgG-HRP (115-035-045) antibodies were provided by Jackson Immunoresearch (West Grove, PA). Dynabeads MyOne Streptavidin Beads (656-01) were purchased from Invitrogen (Carlsbad, CA).

\section{Plasmids}

The retroviral transfer vector pBMN.i.mCherry was constructed by amplifying mCherry from pmCherry-C1 (Clontech, Palo Alto, CA) using the forward primer with extending NcoI site ATCGATGGATCCCCACCATGGTGAGCAAGGG CGAGGAG and reverse primer with extending XhoI site AT GGACGAGCTGTACAAGTAACTCGAGGATCGATC, and inserting it into partially digested pBMN-i-eGFP (Gary Nolan, Stanford University) with NcoI/SalI. The SBP-Citrine construct was a kind gift from Shari Kaiser (Fred Hutchinson Cancer Research Center, Seattle, WA). The construct pBMN.SBP-Citrine.i.mCherry was created by amplifying SBP-Citrine from SBP-Citrine with the forward primer with extending BgIII site TATAGCTAGCAGATCTC-CACCATG
GACGAGAAGACCACCGGC and reverse primer with extending XhoI site ATGGACGAGCTCTATAAAT AACTCGAGTATA, and inserted into pBMN.i.mCherry digested with BamHI/XhoI. The SBP tag was cloned into pcDNA3.1/Zeocin (Invitrogen) by amplifying the SBP sequence with the forward primer TATAGCTAGCAGAT CTCCAC-CATGGACGAGAAGACCACCGGC, which contains a Kozak sequence and a BgIII site and a reverse primer TATACTCGAGTCTAGAG-GATCCGGGCTCCCTCTGGCC CTGGGGG that contains a linker consisting of an XbaI and an XhoI site. This product was ligated into pcDNA3.1/Zeocin using BamHI/XhoI restriction enzymes. pcDNA3.1/Zeocin SBP-CSN1 construct was created by amplifying CSN1 from HeLa cDNA (courtesy of Christopher Glembotski, SDSU) by using a forward primer containing an $\mathrm{XbaI}$ site TATA GGATCCTCTAGACC-GCTGCCGGTTCAGGTGTTT and a reverse primer containing an XhoI site TATACTCGAGTCAC ATGTTGGTGCTCATCCGGG, digesting it with XbaI/XhoI and ligating it into pcDNA3.1/Zeocin. The construct pBMN.SBP-CSN1.i.mCherry was created by digesting pcDNA3.1/Zeocin SBP-CSN1 with BamHI/XhoI and ligating the extracted fragment into pBMN.i.mCherry digested with BamHI/XhoI.

\section{Virus production and transductions}

For the production of MLV based virus, a $10 \mathrm{~cm}^{2}$ plate of Phoenix GP cells at $50 \%$ confluence was transfected with $3 \mu \mathrm{g}$ of the packaging vector (pBMN.SBP-Citrine.i.mCherry or pBMN.SBP-CSN1.i.mCherry) and $3 \mu \mathrm{g}$ of a vector expressing the Envelope glycoprotein of the Vesicular Stomatitis Virus (pCI-VSVg) by mixing the plasmids in $125 \mu \mathrm{L}$ of FCS-free DMEM and $30 \mu \mathrm{g}$ of Polyethylenimine (linear, MW 24000; Polysciences, Inc., Warrington, PA). Media (DMEM with 10\% FCS, Pen-Strep, L-Glutamine) was replaced $24 \mathrm{~h}$ posttransfection and viral supernatant was collected $48 \mathrm{~h}$ after transfection and filtered with 0.45-micron PTFE filters (Pall Corporation, East Hills, NY). The supernatant was used to spin-infect naive SupT1 cells in a six-well plate format. Briefly, viral supernatant was mixed with polybrene $(5 \mu \mathrm{g} / \mathrm{mL}$ final concentrations) and added to the cells, the mixture plated in a six-well plate and spun at $1500 \times g, 32^{\circ} \mathrm{C}$ for $80 \mathrm{~min}$ in a hanging bucket rotors centrifuge (Becton Dickinson, Fullerton, CA). Twenty-four hours postinfection, cells were resuspended in fresh media.

\section{Flow cytometry and sorting}

Flow cyometry and sorting were performed on a BD FACSAria with 405-, 488-, and 633-nm lasers. Data was collected on FACSDiva 6.1.1.

\section{Pull-downs and Western blotting}

Cells were spun and resuspended in modified NP-40 buffer (150 mM NaCl, Tris-Cl 50 mM, 10\% Glycerol, 0.25\% NP-40) supplemented with Complete Protease Inhibitor cocktail (Roche, Indianapolis, IN) at a concentration of $10^{8}$ cells $/ \mathrm{mL}$. After incubation on ice for $30 \mathrm{~min}$, cell lysates were spun at $14,000 \times g$ for $15 \mathrm{~min}$ at $4^{\circ} \mathrm{C}$ and supernatants were transferred to new tubes. The lysates were incubated for $1 \mathrm{~h}$ with $100 \mu \mathrm{L}$ of Dynabeads ${ }^{\circledR}$ MyOne Streptavidin T1 beads (Invitrogen) on a rotating rack at $4^{\circ} \mathrm{C}$. The beads were separated from the sample with a magnetic rack, and the supernatant was 
retained for lysate control. The beads were washed at least five times with $1 \mathrm{~mL}$ lysis buffer, and the bound proteins were eluted with lysis buffer containing $2 \mathrm{nM}$ D-biotin. Lysates and elutes were boiled in SDS sample buffer, run in a $12 \%$ polyacrylamide gel and then transferred to a PVDF membrane. After blocking with 5\% milk in PBST (PBS + 0.05\% Tween 20), the membrane was incubated with the indicated primary antibodies followed by HRP-conjugated secondary antibodies. Proteins were subsequently detected using the Amersham ECL kit.

\section{Preparation of samples for liquid chromatography- mass spectrometry}

Samples were first run in a 12\% Mini-PROTEAN TGX gel (BioRad, Hercules, CA), and the appropriate gel slices containing the sample were sent to Stanford University Mass Spectrometry Core (SUMS) for LC-MS analysis.

\section{Results}

\section{Purification of the CSN holocomplex from SupT1 cells}

In order to purify the CSN complex from T cells we decided to engineer a tagged version of one of the CSN subunits, and express the tagged subunit endogenously. For that purpose, we utilized a 38-amino acid peptide (SBP) shown to have high binding affinity to streptavidin (SA) (Keefe et al., 2001). Previous studies have demonstrated that it is feasible to purify the entire complex through CSN1 (Menon et al., 2005). We thus introduced the SBP sequence at the $\mathrm{N}^{\prime}$ terminus of CSN1, cloned into the pBMN.i.mCherry retroviral vector. In this vector, referred to as $\mathrm{pBMN.SBP-CSN1.i.mCherry,} \mathrm{the} \mathrm{trans-}$ lation of the SBP-CSN1 protein is coupled to the IRESmCherry cassette (i.mCherry) (Fig. 1A). This vector was used to create MLV particles and infect naive SupT1 cells, as previously described (Wolkowicz et al., 2004), which were then
FACS-sorted, clonally selected on the basis of high mCherry expression, and probed with anti-CSN1 antibody to confirm the expression of SBP-CSN1 (Fig .1B-D). As a control, we utilized a similar construct where CSN1 was substituted with Citrine, a modified yellow fluorescent protein chosen to exclude the nonspecific protein-protein interaction of the SBPtag fusion protein. Citrine, a protein of nonmammalian origin, serves here as a stricter control than the empty vector. Total extracts from SBP-Citrine- and -CSN1-expressing cells were then subjected to streptavidin-coated magnetic beads in order to pull down SBP-tagged proteins (Fig. 2).

The eluted fractions were then analyzed for the presence of CSN subunits. Preliminary results (data not shown) indicated that the lysis buffer utilized was too stringent to pull down the entire CSN holocomplex, probably due to the high concentration of the nonionic Nonodet P-40 detergent and the presence of the ionic sodium deoxycholate detergent, both of which can disrupt weak protein-protein interactions. Interestingly, at these high-stringency conditions $(150 \mathrm{mM} \mathrm{NaCl}$, $1.0 \%$ NP-40, $0.5 \%$ sodium deoxycholate, $0.1 \%$ SDS, and $50 \mathrm{mM}$ Tris, $\mathrm{pH} 8.0$ ), CSN subunits $1,2,3$, and 8 could be detected. This finding is consistent with a recent study demonstrating that the CSN subunits form two distinct minicomplexes, one of which is composed of CSN1, 2,3, and 8, that together, assemble into the holocomplex (Sharon et al., 2009). Decreasing the stringency of the lysis buffer by reducing the amount of NP-40 detergent and gentler mechanical lysis resulted in the purification of all eight of the CSN subunits from SupT1 cells, including both known isoforms of CSN7, as confirmed by Western blots and LC-MS (Fig. 3 and Table 1).

\section{Known and predicted binding partners of the CSN isolated from SupT1 cells}

One of the major functions of the CSN is the cleavage of Nedd8 from Cullin proteins (Cope et al., 2002). Prior to LC-MS
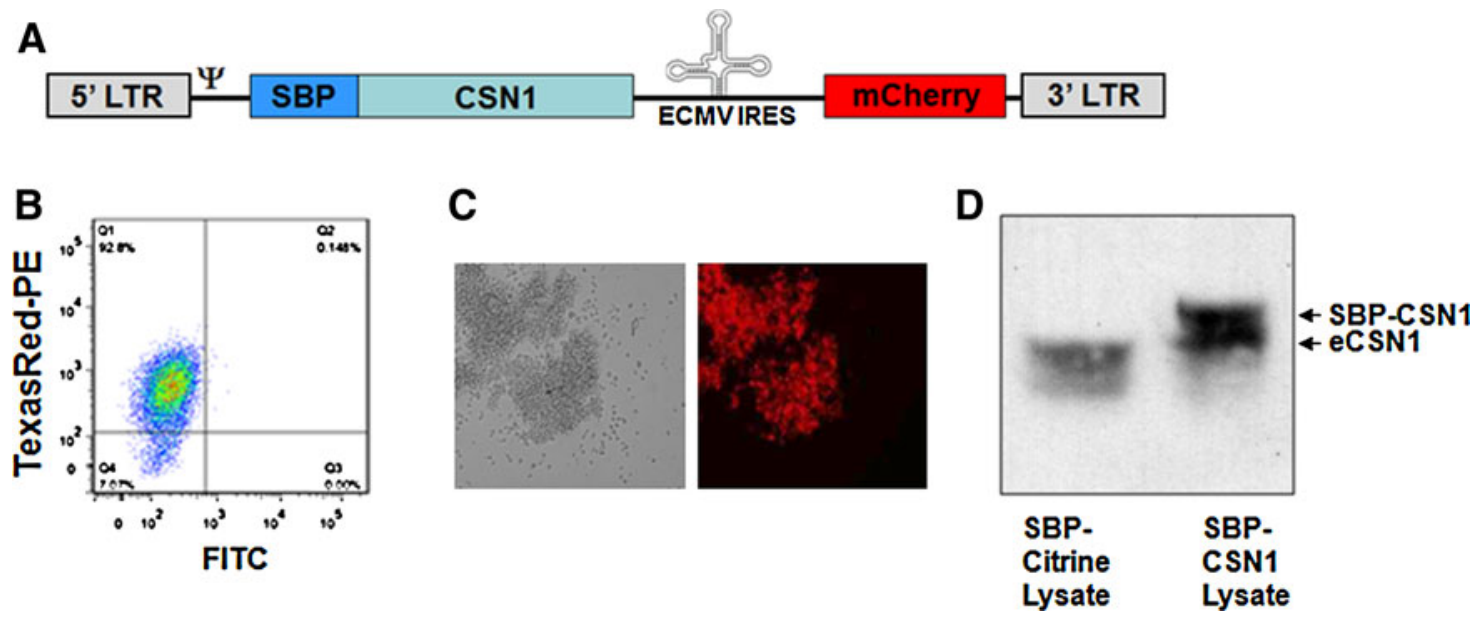

FIG. 1. Establishment of an SBP-CSN1 expressing cell line. (A) Representation of pBMN.SBP-CSN1.i.mCherry, the pBMN.i.mCherry retroviral vector harboring the SBP sequence fused to the $\mathrm{N}^{\prime}$ terminus of CSN subunit 1 . (B) Retroviral particles produced from pBMN.SBP-CSN1.i.mCherry were used to infect naive SupT1 cells. The flow cytometry plot shows the analysis of infected cells sorted on the basis of mCherry expression. (C) Individual sorted cells were grown in 96-well plates, and the brightest clone was selected to establish the SBP-CSN1 cell line. The fluorescence microscopy images of the cell line obtained shows high expression of mCherry. (D) Expression of SBP-CSN1 was confirmed by Western blot analysis. The SBP-Citrine cell line was used as control. The arrows show SBP-CSN1 and endogenous CSN1 (eCSN1) only in the SBP-CSN1 cell line. 


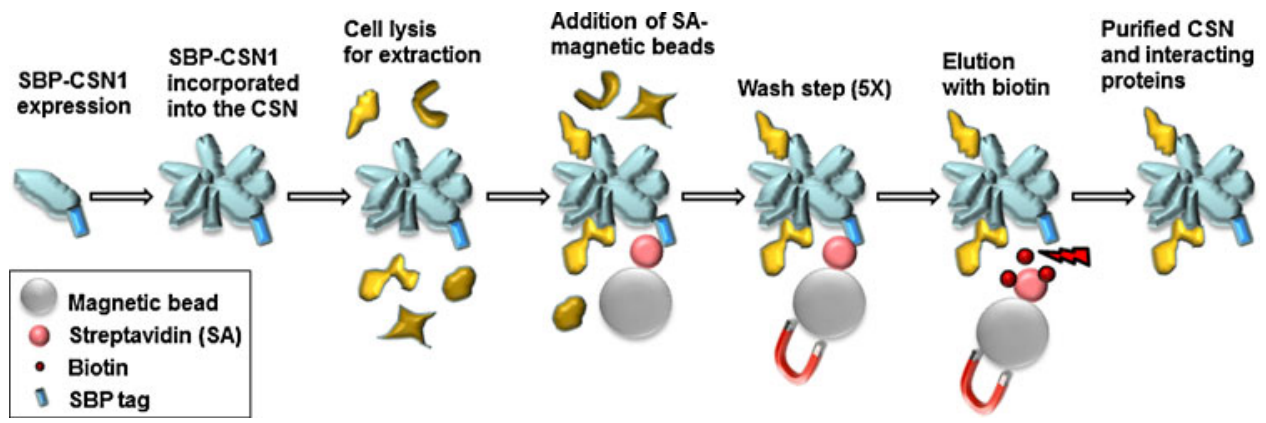

FIG. 2. Schematic representation of the SBP-based pull-down technique for the purification of the CSN and binding partners.

analysis, we probed our eluted fraction with a Nedd8 antibody in order to ascertain if any binding proteins were pulled down along with the CSN holocomplex. Several protein bands were detected, indicating the presence of neddylated proteins in the eluate from the lysate of the SBP-CSN1 cell line (Fig. 4A). We next addressed whether this technique enabled us to isolate the binding partners of the CSN, either as previously described in literature or predicted by bioinformatics tools in silico (Table 2). The eluted fractions from SBP-CSN1and SBP-Citrine whole-cell lysates were analyzed by LC-MS at the Stanford University Mass Spectrometry facility. Hits from the SBP-Citrine pull-down were used to eliminate nonspecific binding proteins. A total of 355 proteins were identified as putative CSN-binding partners. Out of 355, 78 showed an $80 \%$ or greater protein identification probability, as determined by the probability of peptide identification and the number of hits identified per protein (Supplementary Tables 1 and 2). The specific hits listed in Table 2 (16 in total), represent hits that have been characterized in other studies, predicted to interact with the CSN in silico or confirmed in our manuscript. From the putative CSN-binding proteins, we identified two Cullin proteins, Cullin 3 and Cullin 4B, in addition to Cullin 4A, which we detected by Western blot (Olma et al., 2009) (Fig. 4B). We have also identified CAND1 and DDB1, a subunit of the damaged-DNA binding protein DDB. These are two of the proteins known to interact with Cullin 4 during the process of ubiquitination of target proteins by the CSN (Bondar et al., 2006). Another probable hit was Ubiquitin ligase E2N (UBE2N), a human analog of Saccharomyces cerevisiae ubiquitin ligase Ubc13. The interaction between $\mathrm{E} 2 \mathrm{~N}$ and the CSN has previously been predicted by genome-scale genetic interaction maps, but never confirmed in either yeast or
A

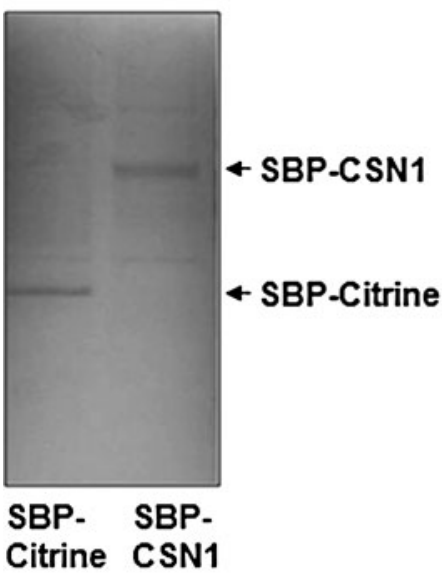

B

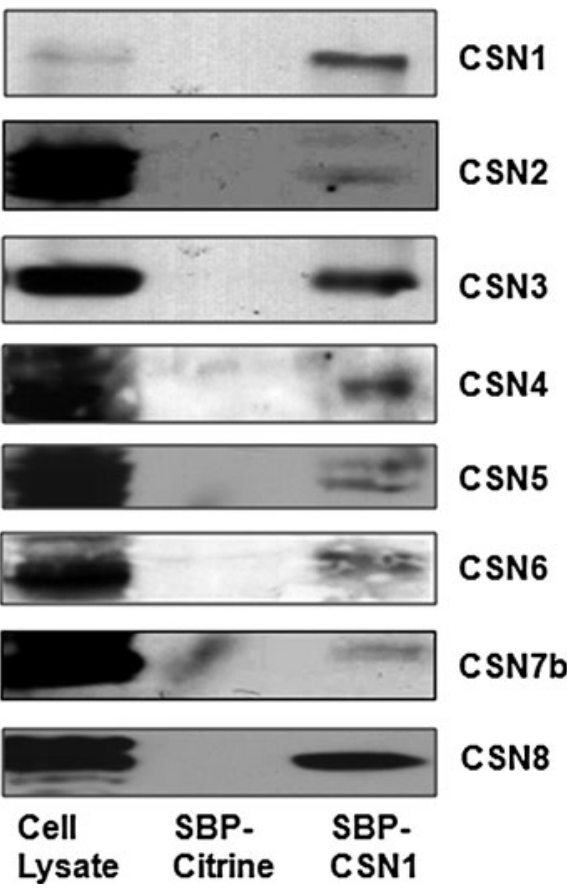

FIG. 3. Purification of the CSN from T cells. (A) Coomassie stain of the eluted fractions isolated from SBP-Citrine control and SBP-CSN1 cell line lysates. (B) Confirmation by Western blot of the presence of all subunits of the CSN in the eluted fractions from the SBP-CSN1 lysate. 
Table 1. Peptides of the CSN Subunits Identified by LC-MS

\begin{tabular}{|c|c|c|c|}
\hline Subunit & Number of peptides & Percentage of Amino Acids Identified & Representative Peptide Sequence(s) \\
\hline CSN1 & 15 & $42 \%$ & $\begin{array}{l}\text { (R)GHDDLGDHYLDCGDLSNALK(C) } \\
\text { (R)ALIQYFSPYVSADMHR(M) } \\
\text { (K)MLDEMKDNLLLDMYLAPHVR(T) }\end{array}$ \\
\hline CSN2 & 6 & $17 \%$ & $\begin{array}{l}\text { (K)SINSILDYISTSK(Q) } \\
\text { (K)WTNQLNSLNQAVVSK(L) } \\
\text { (K)SGINPFDSQEAK(P) }\end{array}$ \\
\hline CSN3 & 7 & $25 \%$ & $\begin{array}{l}\text { (R)FIKPLSNAYHELAQVYSTNNPSELR(N) } \\
\text { (K)YVLHMIEDGEIFASINQK(D) } \\
\text { (R)VQLSGPQEAEK(Y) }\end{array}$ \\
\hline CSN4 & 8 & $29 \%$ & $\begin{array}{l}\text { (K)LYNNITFEELGALLEIPAAK(A) } \\
\text { (R)NAAQVLVGIPLETGQK(Q) } \\
\text { (K)IASQMITEGR(M) }\end{array}$ \\
\hline CSN5 & 4 & $18 \%$ & $\begin{array}{l}\text { (R)VNAQAAAYEYMAAYIENAK(Q) } \\
\text { (K)GYKPPDEGPSEYQTIPLNK(I) } \\
\text { (R)SGGNLEVMGLMLGK(V) }\end{array}$ \\
\hline CSN6 & 3 & $16 \%$ & $\begin{array}{l}\text { (K)FNVLYDR(Q) } \\
\text { (R)SQEGRPVQVIGALIGK(Q) } \\
\text { (R)NIEVMNSFELLSHTVEEKIIIDKEYYYTK(E) }\end{array}$ \\
\hline CSN7a & 1 & $3.3 \%$ & (R)LEVDYSIGR(D) \\
\hline CSN7b & 3 & $18 \%$ & $\begin{array}{l}\text { (K)ATASSSAQEMEQQLAER(E) } \\
\text { (R)TQQQVEAEVTNIK(K) } \\
\text { (R)ELEDLIIEAVYTDIIQGK(L) }\end{array}$ \\
\hline CSN8 & 7 & $62 \%$ & $\begin{array}{l}\text { (K)FIPLSEPAPVPPIPNEQQLAR(L) } \\
\text { (K)SANSELGGIWSVGQR(I) } \\
\text { (R)AFALVSQAYTSIIADDFAAFVGLPVEEAVK(G) }\end{array}$ \\
\hline
\end{tabular}

human cells (Huttenhower et al., 2009). Other members of the ubiquitination machinery included Ubiquitin protein ligase E3A (UBE3A) and Isoform 4 of Ubiquitin protein ligase (UBR4).

Several protein hits from our pulldown corresponded with predictions made by the Human Protein-Protein Prediction
Interaction database (PIP), a bioinformatic tool that predicts the likelihood of interaction between two proteins (McDowall et al., 2009). These included Long Isoform of 14-3-3 protein beta/alpha (YWHAB, interaction score of 5.28) and Eukaryotic translation initiation factor 5A-2 (EIF5A2, interaction
A

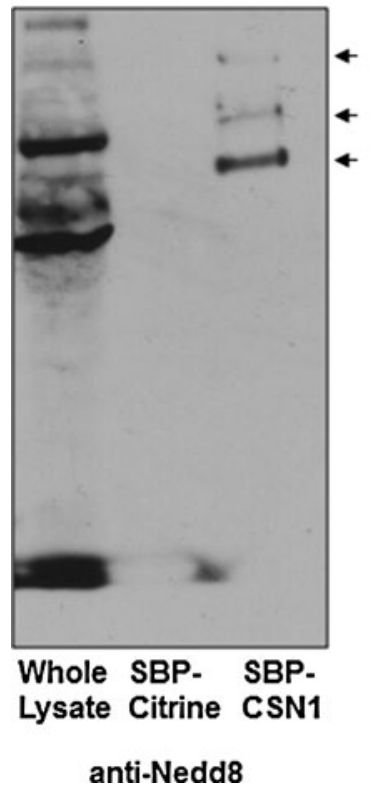

B

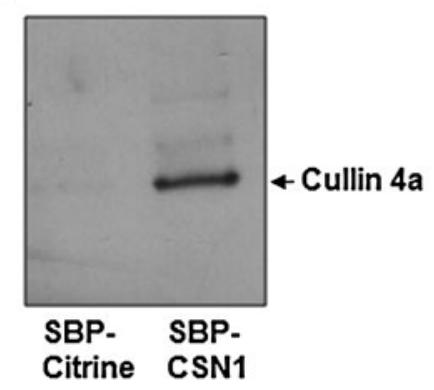

C

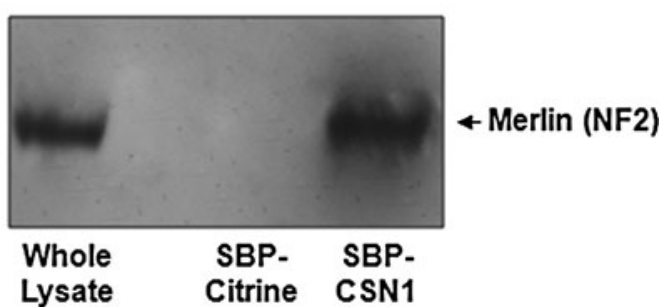

FIG. 4. Western blot analysis of SBP-Citrine and SBP-CSN1 cell lysates to confirm CSN binding partners. (A) Cell line eluates were probed with anti-Nedd8 antibody to show the interaction of the CSN with neddylated proteins (three arrows) in the SBP-CSN1 lysate but not in the SBP-Citrine control. Anti-Cullin 4A (B) and anti-Merlin (NF2) (C) antibodies were used to confirm the interaction of the CSN with Cullin 4A and Merlin. 
Table 2. Peptides of Proteins Binding to the CSN Identified by LC-MS

\begin{tabular}{|c|c|c|c|c|}
\hline Accession number & Protein & Protein name & Peptide sequence & Peptide prob. \\
\hline IPI00179057.6 & CUL4B & Cullin- $4 \mathrm{~B}^{\mathrm{a}}$ & (K)TIDGILLLIER(E) & $90 \%$ \\
\hline IPI00014312.1 & CUL3 & Cullin- $3^{\mathrm{a}}$ & (R)SPEYLSLFIDDK(L) & $95 \%$ \\
\hline IPI00100160.3 & CAND1 & $\begin{array}{l}\text { Cullin-associated NEDD8- } \\
\text { dissociated protein }\end{array}$ & (K)LGTLSALDILIK(N) & $95 \%$ \\
\hline IPI00293464.5 & DDB1 & DNA Damage-binding protein ${ }^{\mathrm{a}, \mathrm{c}}$ & (R)LFMLLLEK(E) & $85 \%$ \\
\hline IPI00299155.5 & PSMA4 & Proteasome subunit alpha type- 4 & (R)TTIFSPEGR(L) & $95 \%$ \\
\hline IPI00028004.2 & PSMB3 & Proteasome subunit beta type- 3 & (R)LYIGLAGLATDVQTVAQR(L) & $95 \%$ \\
\hline IPI00479722.2 & PSME1 & Proteasome activator complex subunit 1 & (K)APLDIPVPDPVK(E) & $95 \%$ \\
\hline IPI00219622.3 & PSMA2 & Proteasome subunit alpha type- 2 & (K)LVQIEYALAAVAGGAPSVGIK(A) & $95 \%$ \\
\hline IPI00216318.5 & YWHAB & $\begin{array}{l}\text { Long Isoform of } 14-3-3 \\
\text { protein beta/alpha }{ }^{b}\end{array}$ & (K)TAFDEAIAELDTLNEESYK(D) & $95 \%$ \\
\hline IPI00006935.3 & EIF5A2 & $\begin{array}{l}\text { Eukaryotic translation } \\
\text { initiation factor } 5 \mathrm{~A}-2^{\mathrm{b}}\end{array}$ & (K)VHLVGIDIFTGK(K) & $91 \%$ \\
\hline IPI00385649.2 & LRCH3 & $\begin{array}{l}\text { Isoform } 2 \text { of Leucine-rich repeat } \\
\text { and calponin homology } \\
\text { domain-containing protein } 3^{\mathrm{a}}\end{array}$ & (Q)YEEEKIRTK(Q) & $95 \%$ \\
\hline IPI00003949.1 & UBE2N & Ubiquitin-conjugating enzyme E2 N & (R)YFHVVIAGPQDSPFEGGTFK(L) & $95 \%$ \\
\hline IPI00640981.3 & UBR4 & $\begin{array}{l}\text { Isoform } 4 \text { of E3 ubiquitin-protein } \\
\text { ligase UBR } 4\end{array}$ & (R)NYLSVSLSEHHMATLASIIK(E)' & $90 \%$ \\
\hline IPI00329038.5 & CDK5RAP2 & $\begin{array}{l}\text { Isoform } 1 \text { of CDK5 regulatory } \\
\text { subunit-associated protein } 2\end{array}$ & (R)DKQKENDK(L) & $89 \%$ \\
\hline IPI00021266.1 & RPL23A & 60S Ribosomal Protein $23 \mathrm{~A}^{\mathrm{c}}$ & (K)IEDNNTLVFIVDVK(A) & $95 \%$ \\
\hline IPI00220308.1 & NF2 & Isoform 2 of Merlin & (K)LLAQKAAEAEQEMQR(I) & $89 \%$ \\
\hline
\end{tabular}

aproteins previously described to interact with the CSN.

${ }^{b}$ Proteins predicted to interact with the CSN by PIPs (Human Protein-Protein Interaction Prediction).

' Proteins predicted to interact with the CSN by BioGRID ${ }^{3.1}$.

score of 2.68). The CSN has previously been shown to associate with the 26S Proteasome, most likely in order to direct very specific proteolysis (Huang et al., 2005). We indeed were able to identify several of the proteosomal subunits, including Proteasome subunit alpha type-2 (PSMA2) and 4 (PSMA4), beta type-3 (PSMB3), and Proteasome activator complex subunit-1 (PSME1). Interestingly, the BioGrid 3.1 protein interaction database (Stark et al., 2011) and the Drosophila protein interaction map based on a yeast two-hybrid screen (Giot et al., 2003) hinted at the interaction between the Ribosomal protein L23A and CSN3, a high-probability hit obtained in our pulldown (Table 2).

\section{Novel CSN binding partner Neurofibromin 2}

One of the strong hits identified in our pulldown was Neurofibromin 2 (Merlin). Merlin is a 70-kDa protein related to proteins that anchor the actin cytoskeleton to specific membrane proteins, and appears to function as a tumor suppressor. A recent study determined that Merlin is targeted to the Roc1-CUL4A-DDB1 E3 ligase complex for degradation by Vpr-binding protein (VprBP) (Huang and Chen, 2008). We further confirmed the presence of Merlin in the SBP-CSN1 eluate by Western blotting (Fig. 4C).

\section{Discussion}

Although the COP9 Signalosome plays a major role in many vital cellular functions, few proteomic studies analyzing its binding partners in human cells have been performed, and none in T cells. In this work, we used retroviral technology to efficiently deliver the SBP-tagged CSN1 subunit into a $\mathrm{T}$-cell line. The SBP-tag provides a fast, efficient, and relatively specific one-step method for the isolation of protein complexes, as demonstrated here with the CSN. Fang et al. (2008) successfully purified the CSN holocomplex from fibroblasts utilizing a long tag of around 110 amino acids in length. This tag included two six-Histidine residue sequences, a 75 amino acid-long sequence as in vivo substrate for biotinylation, and a 29 amino acid-long Tobacco Etching Virus protease cleavage site with linkers. The SBP-tag is only 38 amino acids-long and does not rely on the efficiency with which the biotinylation process occurs in the cell. Furthermore, in the SBP-tag system, biotin is used as competitor at the time of purification and purification does not rely on the activity of a protease. Using the SBP-tagged CSN1 subunit expressed endogenously, we were able to recover all eight subunits of the CSN through the pull down, demonstrating that tagging the CSN subunit did not disrupt its ability to interact with the rest of the subunits and form the CSN holocomplex. Previous studies for the purification of the CSN holocomplex in human cells were performed in fibroblasts. Here we have successfully purified the complex from $\mathrm{T}$ cells, a nonadherent cell type with very different biological characteristics and functionalities, such as those related to immune activation. Although SupT1 cells are not primary cells, they are easily activated by many physiological and nonphysiological factors, and infectable by viruses such as HIV-1. This is not to say that parallel experiments should not be performed in primary $\mathrm{T}$ cells, where similar results should nonetheless be expected. Furthermore, combining this approach with LC-MS yielded a large number of potential CSN binding partners. These included previously documented members of the ubiquitin-ligase machinery, such as CAND1, DDB1, and various Cullins, as well as novel ubiquitin ligases UBR4, UBE3A, and UBE2N. In addition, we 
were also able to detect several protein components of the $26 \mathrm{~S}$ Proteasome, a finding that is consistent with the model of the CSN-Proteasome supercomplex, where the CSN directly interacts with the barrel of the 26S Proteasome to specifically degrade protein targets (Huang et al., 2005). Importantly, we were able to detect several proteins predicted by in silico tools such as BioGrid 3.1 and PIP, such as YWHAB, EIF5A2, and L23A. The nature of this interaction is unknown. Nevertheless, the fact that one of the main roles of the CSN in involves phosphorylation via CSN-associated kinases and the fact that both YWHAB and L23A are known to be phosphorylated during cellular processes reassures us that purification of these targets may have a significant biological role (Dai et al., 2004; Liu et al., 2007).

Interestingly, another putative novel binding partner of the CSN with no known relation to the proteasomal and/or ubiquitination machineries was Neurofibromin 2 (Merlin). In a recent study, it was found that the cellular levels of Merlin are controlled by the HIV-1 Vpr-Binding Protein, a protein known to act as a substrate-recognition subunit of the ROC1CUL4A-DDB1 ubiquitin ligase complex and which appears to direct Merlin for degradation via ubiquitination (Huang and Chen, 2008; Wen et al., 2007). The finding that Merlin is associated with the COP9 Signalosome is consistent with the model describing the control of Merlin expression, as one of the functions of the CSN is to deneddylate Cullin proteins, including CUL4A, in order to negatively regulate the ubiquitination of their targets (Deshaies, 1999).

The identification of all eight subunits of the CSN (including both isoforms of CSN7, as demonstrated by LC-MS and Western blotting) and a number of its binding partners from $T$ cells, including Merlin, demonstrates that the purification method through intracellularly expressed SBP tag is a robust method for protein-binding discovery. A large number of hits were found to be nonspecific and bind to the SBP tag, streptavidin, and/or beads, particularly mitochondrial proteins. The method described here for the purification of the CSN may thus not be appropriate for targeting protein complexes such as those associated with the mitochondria, emphasizing the importance of a control, SBP-Citrine in our experiments, for determining specific binding. The advantages of using the SBP tag include the relatively rapid pulldown procedure $(20 \mathrm{~min}$ incubation for most protein isolations combined with rapid magnetic rack-based washes) and high specificity of streptavidin to SBP compared to immunoprecipitation methods involving antibodies. This makes the method an attractive alternative for sample preparation for LC-MS analysis.

Purification of the CSN holocomplex together with its binding partners from a natural environment such as a $\mathrm{T}$ cell can greatly facilitate the study of the CSN and its interactions as well as the regulation of its binding partners under different conditions, such as T cell activation and HIV-1 infection.

\section{Acknowledgments}

R.W. was supported by the California HIV / AIDS Research Grant Program (CHRP) ID09-SDSUF-023 \#163058, and the California State University Program for Education and Research in Biotechnology (CSUPERB). The authors thank Dr. Shari Kaiser (Institute for Systems Biology, Seattle, WA) for providing the SBP-Citrine construct, Brett Hilton from the SDSU Fluorescence Activated Cell Sorting facility, the Stan- ford University Mass Spectrometry Facility, and the San Diego Chapter of the ARCS (Achievement Rewards for College Scientists) Foundation, who supported A.S.

\section{Author Disclosure Statement}

The authors declare that no conflicting financial interests exist.

\section{References}

Bech-Otschir, D., Kraft, R., Huang, X., et al. (2001). COP9 signalosome-specific phosphorylation targets p53 to degradation by the ubiquitin system. EMBO J 20, 1630-1639.

Bondar, T., Kalinina, A., Khair, L., et al. (2006). Cul4A and DDB1 associate with Skp2 to target p27Kip1 for proteolysis involving the COP9 Signalosome. Mol Cell Biol 26, 2531-2539.

Claret, F.X., Hibi, M., Dhut, S., et al. (1996). A new group of conserved coactivators that increase the specificity of AP-1 transcription factors. Nature 383, 453-457.

Cope, G.A., Suh, G.S.B., Aravind, L., et al. (2002). Role of predicted metalloprotease motif of Jab1/Csn5 in cleavage of Nedd8 from cul1. Science 298, 608-611.

Dai, M.-S., Zeng, S.X., Jin, Y., et al. (2004). Ribosomal protein L23 activates p53 by inhibiting MDM2 function in response to ribosomal perturbation but not to translation inhibition. Mol Cell Biol 24, 7654-7668.

Deshaies, R.J. (1999). SCF and cullin/ring H2-based ubiquitin ligases. Annu Rev Cell Dev Biol 15, 435-467.

Fang, L., Wang, X., Yamoah, K., et al. (2008). Characterization of the human COP9 signalosome complex using affinity purification and mass spectrometry. J Proteome Res 7, 4914-4925.

Giot, L., Bader, J.S., Brouwer, C., et al. (2003). A protein interaction map of Drosophila melanogaster. Science 302, 1727-1736.

Huang, J., and Chen, J. (2008). VprBP targets Merlin to the Roc1Cul4A-DDB1 E3 ligase complex for degradation. Oncogene 27, 4056-4064.

Huang, X., Hetfeld, B.K.J., Seifert, U., et al. (2005). Consequences of COP9 signalosome and $26 \mathrm{~S}$ proteasome interaction. FEBS J. 272, 3909-3917.

Huttenhower, C., Haley, E.M., Hibbs, M.A., et al. (2009). Exploring the human genome with functional maps. Genome Res 19, 1093-1106.

Karniol, B., Yahalom, A., Kwok, S., et al. (1998). The Arabidopsis homologue of an eIF3 complex subunit associates with the COP9 complex. FEBS Lett 439, 173-179.

Keefe, A.D., Wilson, D.S., Seelig, B., et al. (2001). One-step purification of recombinant proteins using a nanomolar-affinity streptavidin-binding peptide, the SBP-tag. Protein Expression Purif 23, 440-446.

Kleemann, R., Hausser, A., Geiger, G., et al. (2000). Intracellular action of the cytokine MIF to modulate AP-1 activity and the cell cycle through Jab1. Nature 408, 211-216.

Liu, Y., Ross, J.F., Bodine, P.V.N., et al. (2007). Homodimerization of Ror2 tyrosine kinase receptor induces 14-3-3(beta) phosphorylation and promotes osteoblast differentiation and bone formation. Mol Endocrinol 21, 3050-3061.

McDowall, M.D., Scott, M.S., and Barton, G.J. (2009). PIPs: human protein-protein interaction prediction database. Nucleic Acids Res 37, D651-D656.

Menon, S., Rubio, V., Wang, X., et al. (2005). Purification of the COP9 signalosome from porcine spleen, human cell lines, and Arabidopsis thaliana plants. Methods Enzymol 398, 468-481.

Miyauchi, Y., Kato, M., Tokunaga, F., et al. (2008). The COP9/ signalosome increases the efficiency of von Hippel-Lindau 
protein ubiquitin ligase-mediated hypoxia-inducible factoralpha ubiquitination. J Biol Chem 283, 16622-16631.

Morimoto, M., Nishida, T., Honda, R., et al. (2000). Modification of Cullin-1 by ubiquitin-like protein Nedd8 enhances the activity of SCFskp2 toward p27kip1. Biochem Biophys Res Commun 270, 1093-1096.

Olma, M.H., Roy, M., Bihan, T. Le, et al. (2009). An interaction network of the mammalian COP9 signalosome identifies Dda1 as a core subunit of multiple Cul4-based E3 ligases. J Cell Sci 122, 1035-1044.

Read, M.A., Brownell, J.E., Gladysheva, T.B., et al. (2000). Nedd8 modification of Cul-1 activates SCFbeta TrCP-dependent ubiquitination of Ikappa Balpha. Mol Cell Biol 20, 2326-2333.

Schwechheimer, C. (2004). The COP9 signalosome (CSN): an evolutionary conserved proteolysis regulator in eukaryotic development. Biochim Biophys Acta 1695, 45-54.

Seeger, M., Kraft, R, Ferrell, K., et al. (1998). A novel protein complex involved in signal transduction possessing similarities to $26 \mathrm{~S}$ proteasome subunits. FASEB J 12, 469-478.

Sharon, M., Mao, H., Boeri erba, E., et al. (2009). Symmetrical modularity of the COP9 signalosome complex suggests its multifunctionality. Structure 17, 31-40.

Stark, C., Breitkreutz, B.-J., Chatr-Aryamontri, A., et al. (2011). The BioGRID Interaction Database: 2011 update. Nucleic Acids Res 39, D698-D704.

Uhle, S., Medalia, O., Waldron, R., et al. (2003). Protein kinase CK2 and protein kinase D are associated with the COP9 signalosome. EMBO J 22, 1302-1312.
Wei, N., and Deng, X.W. (1992). COP9: a new genetic locus involved in light-regulated development and gene expression in Arabidopsis. Plant Cell 4, 1507-1518.

Wei, N., and Deng, X.W. (2003). The COP9 signalosome. Annu Rev Cell Dev Biol 19, 261-286.

Wen, X., Duus, K.M., Friedrich, T.D., and Noronha, C.M.C. de. (2007). The HIV1 protein Vpr acts to promote G2 cell cycle arrest by engaging a DDB1 and Cullin4A-containing ubiquitin ligase complex using VprBP/DCAF1 as an adaptor. J Biol Chem 282, 27046-27057.

Wolkowicz, R., Nolan, G.P., and Curran, M.A. (2004). Lentiviral vectors for the delivery of DNA into mammalian cells. Methods Mol Biol 246, 391-411.

Zheng, J., Yang, X., Harrell, J.M., et al. (2002). CAND1 binds to unneddylated CUL1 and regulates the formation of SCF ubiquitin E3 ligase complex. Mol Cell 10, 1519-1526.

Address correspondence to: Roland Wolkowicz Department of Biology San Diego State University North Life Sciences Room 304, 5500 Campanile Drive San Diego, CA 92182

E-mail: roland@sciences.sdsu.edu 